\title{
Introduction to the contents of issue 52:3
}

\author{
Axel Ruhe
}

Published online: 28 August 2012

(C) Springer Science + Business Media B.V. 2012

BIT has emerged as one of the primary publication channels for stochastic differential equations, SDE, a fruitful way to study a system where dynamic and random influences have to be considered simultaneously. We are very proud that! In this issue, we have three papers on the numerical treatment of SDEs. In the first one, Jamie Alcock and Kevin Burrage treat a stiff SDE by the quasi implicit balanced method and show how the stability advantage of implicit methods can be brought over to the stochastic case. A method that is strongly stable of order 1.0 is developed and tested on an application, the Sagirow's satellite problem. The second to last paper in this issue, written by Xiaojie Wang, Siqing Gan, and Desheng Wang, treats a related problem. It studies a family of fully implicit Milstein methods of strong stability order 1.0, and illustrate its behavior on two specially designed test problems. In another paper, P.A. Cioica, S. Dahlke, N. Döhring, S. Kinzel, F. Lindner, T. Raasch, K. Ritter, and R.L. Schilling develop a wavelet algorithm for a Poisson equation with a random right hand side. Convergence in a Besov space setting is established, and the regularity is illustrated in a numerical example.

BIT has also become even more present in numerical treatment of partial differential equations, PDE! In this issue, Sören Bartels and Patrick Schreier develop a reversible bisection refinement strategy for simplicial finite element meshes, which allows easy coarsening when a local error criterion signals that this is allowable. The success of an adaptive mesh strategy is crucially dependent on this nontrivial geometric problem. The strategy is illustrated on a problem with corner singularities and one with a moving interface. In another contribution, Björn Engquist, Jon Häggblad, and Olof Runborg study how to apply the well established finite difference time domain,

\footnotetext{
A. Ruhe (凶)

School of Computer Science and Communication (CSC), Royal Institute of Technology (KTH), 10044, Stockholm, Sweden

e-mail: ruhe@kth.se
} 
FDTD, or Yee scheme for electromagnetic computations, to problems with curved boundaries. They keep the structured overlapping grids, but change the coefficients for points at the boundary. These modifications are simple to include in an existing code. A rigorous $L_{2}$ stability analysis is given. Ralf Hiptmair and Shipeng Mao study a multilevel mesh refinement for a boundary element space and prove its stability.

Two papers consider methods for time stepping in dynamic systems. In the first one Mohammad Shakourifar and Wayne Enright study a piecewise polynomial collocation method for a Volterra integro differential equation with delay, and show how to make it superconvergent in all points. The method is illustrated on a typical predator prey application. In the last paper of this issue, Xinyuan Wu, Bin Wang, and Jianlin Xia study multidimensional exponential fitting modified Runge-Kutta-Nyström methods for systems of oscillatory second order differential equations. The preservation of symplecticity is given special attention.

Three papers investigate linear algebra aspects of algorithms, another mainstay of BIT. In the first one, Per Bergström, Ove Edlund, and Inge Söderkvist consider how to compute the parameters of a non-uniform rational B-spline, NURBS, curve, fitting a set of measured points in the least squares sense. A Gauss Newton algorithm with separated variables is developed, and its convergence is established. In the second linear algebra paper, Volker Grimm describes a resolvent, or rational, Krylov algorithm to compute the exponential function of a matrix, applied to a vector. It is used in an exponentially fitted time stepping method for a PDE, discretized either with a pseudospectral approximation, finite differences or finite elements. Finally Gérard Meurant continues his long study of Krylov iterations, and specially the ubiquitous general minimal residual algorithm, GMRES. It has became the standard tool, when you need to solve a large nonsymmetric linear system but do not have any special information available. He shows the intricate influence on the convergence behavior of properties of the matrix, its spectrum and the starting vector.

Finally, one paper concerns particulars of an approximation problem. Georg Muntingh develops an algorithm to compute divided differences of a multivariate function that is implicitly defined by a nonlinear equation. The formula involves a sum over a combinatorial structure whose elements can be viewed either as polygonal partitions or as plane trees. It is shown, how the well known Faá di Bruno formula for derivatives will occur when the points coalesce.

I wish you all a pleasant and rewarding read,

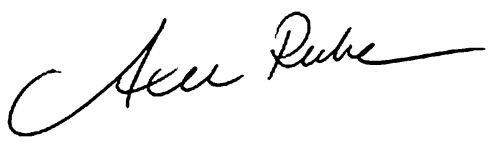

Axel Ruhe 